\title{
Communication Network Availability Predictions Based on Measurement Data
}

\author{
JOHN D. SPRAGINS, MEMBER, IEEE, JAMES D. MARKOV, M. W. DOSS, STEPHANIE A. MITCHELL, AND \\ DAVID C.SQUIRE
}

\begin{abstract}
Published data describing the probability distributions of data communication system reliability parameters such as availability are very sparse. This paper describes a set of data collected in order to model communication line availability by monitoring operational communication systems. Probability distribution functions for single line availabilities and for the durations of failures are given. A heuristic model for estimating availabilities for more complex systems is presented along with computer simulation data validating the accuracy of the model. The results presented make possible more realistic availability predictions for common types of networks than have previously been computable.
\end{abstract}

\section{INTRODUCTION}

A VARIETY of designs for distributed function computer communication systems have been developed since the late 1960 's, with a wide variety of such systems currently being developed and installed in countries around the world. One of the key concerns in the design of such systems is the reliability of telephone systems for transmitting data, since the great majority of the data communication links currently in use are telephone links.

Reliability of telephone systems is not a new concern, but the new systems being designed, with their emphasis on realtime operation, have forced reliability to be considered in a different way. Prior to this time, a great deal of effort was spent on understanding bit error characteristics of telephone circuits. This information has allowed various types of error detecting and correcting methods, and corresponding data transmission protocols, to be developed to ensure almost error-free transmission of data. As these error-handling techniques become more successful, long-term communication line outages are becoming the dominant factor limiting data communication reliability. In contrast to short-term bit errors, the new concern is over failures of telephone lines that last minutes or hours.

A considerable amount of very fine work has been done to understand bit error rates. Examples of this work can be found

Paper approved by the Editor for Computer Communication of the IEEE Communications Society for publication without oral presentation. Manuscript received February 26, 1980; revised January 30, 1981. This work was based in part upon work supported by the National Science Foundation under Grant ENG 78-03384 and Tektronix, Inc. through its graduate education program.

J. D. Spragins is with the Department of Electrical Engineering, Clemson University, Clemson, SC 29631.

J. D. Markov and M. W. Doss are with the IBM Corporation, Research Triangle Park, NC 27709.

S. A. Mitchell is with the IBM Corporation, Winston-Salem, NC 27105.

D. C. Squire is with Tektronix, Inc., Beaverton, OR 97077. in [1]-[4]. This paper addresses the concern over failures lasting longer than $1 \mathrm{~min}$ to augment the information about bit error rates and provide an overall reliability model for a transmission line. Longer term outages such as those motivating this study have received far less attention than bit errors, so no references discussing long-term outages in a manner comparable to [1]-[4] have been published. This paper contains more detailed measurements for long-term outages than have previously been published.

The primary reliability measure studied here is system availability, which can be defined as the probability the system is operational at a randomly chosen instant of time (normally interpreted as the instant when a user wishes to use the system). An equivalent measure is system unavailability, one minus availability, which gives the probability that the system is not operational at a randomly chosen instant of time. Most of the plots given here are distribution functions for unavailability.

The measurement data included here were obtained for systems of the form indicated in Fig. 1. Two logical machines are interconnected by a communication line provided by a PTT or communications common carrier in the manner shown. The distance between the two machines is variable from a mile or so up to thousands of miles. Furthermore, the routing of the line between the two machines is normally unknown to the customer. (The actual routing distance may be several times the airline distance between the end points [5].) The two machines may be in any of a variety of countries or even in different countries.

\section{MEASUREMENT DATA}

\section{Sources of Data}

The next few sections of this paper summarize a set of data which can be used to characterize communication lines. To better appreciate these results, it is important to understand the sources of the data. Despite several obvious weaknesses in the sampling process, ascribed below, the data obtained have proven to be remarkably consistent. Important parameters such as the mean and the 90th percentile of availability distributions (the two primary parameters used in the theoretical analysis which follows) tended to differ for different systems observed by only a small fraction of a percent.

Most of the data presented here come from teleprocessing (TP) system logs, which have been compiled by users of TP systems for their own purposes. In some cases, copies of the actual $\operatorname{logs}$ were obtained and detailed data were available. 


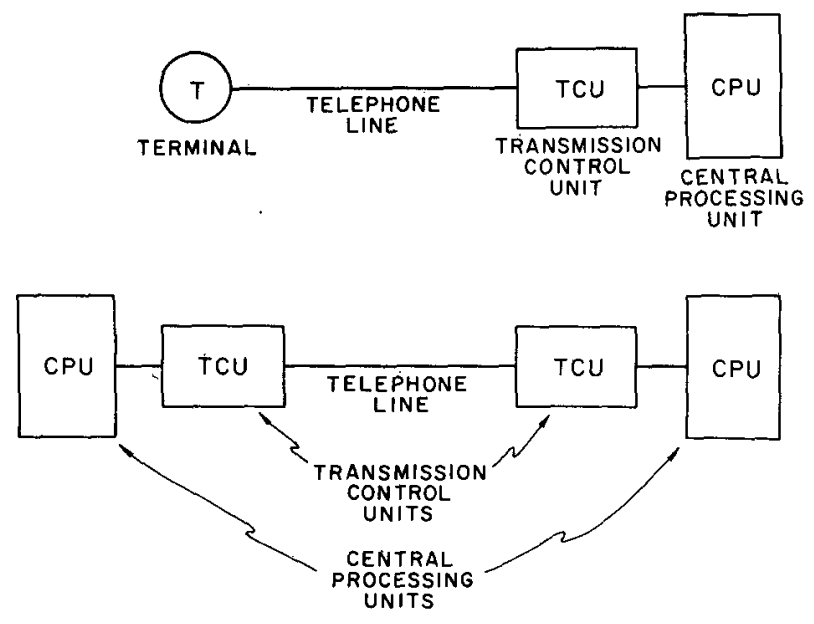

Fig. 1. System description.

In others, only reduced summaries were obtained. The data were compiled for a total of 118 multidrop lines, with 415 branches or drops, in North America and Europe. Some lines were configured with multiple branches or drops, while others were point to point. For clarity, the information here is presented on a per-drop basis, with each drop called a branch. The observation times for lines ranged from three months to three years, with most systems (about 90 percent) monitored for at least six months of continuous operation. (While a considerable amount of information with shorter observation periods was obtained, this was only used for reasonableness checks on the results.) A total of 1233 line-months and 3799 branchmonths of observations are included in the data. (This excludes a small sample of 11 high-speed, $50 \mathrm{kbit} / \mathrm{s}$ lines, used for a curve examining speed variations given below, but otherwise excluded from the summary data.) The earliest data reported date back to 1965 , with additional data collected over a ten year period since then.

A list of the major categories of lines considered, and the numbers of branches (drops) in each category, is given in Table I.

The data presented here should not be interpreted as being from an ideal statistical sampling process. The amount of data for some classes of lines is not really adequate, and the sources of data have not been properly distributed over the various classes of TP systems or over various geographical regions. Nevertheless, it has practical value and is more comprehensive than any similar data published previously. In addition, the TP system logs used were not all recorded under the same conditions. In particular, the definition of a failure was not clearly established; a system or subsystem was defined to have failed when the persons maintaining the log for that system recorded a failure, with various operators displaying different degrees of tolerance for the level of system degradation which they considered to merit recording. In addition, it was not always possible to fully separate out the effects of line or branch failures from those of failures of other equipment such as modems, terminal gear, etc. Although the data given are felt to be dominated by telephone line failures, other failures doubtless enter in also. Unfortunately, the data do not allow the effects of those other failures to be quantified.
TABLE I

LINE CLASSES STUDIED; SEE SECTIONS ON SPEED VARIATIONS AND COUNTRY AND DISTANCE VARIATIONS FOR DEFINITIONS OF CLASSES

\begin{tabular}{lc}
\hline Class & Number of Branches \\
\hline Short & 14 \\
Long (national) & 381 \\
International & 20 \\
North America & 271 \\
Europe & 144 \\
Low Speed & 87 \\
Medium Speed & 328 \\
High Speed & 11 \\
Total (all save high speed) & 415 \\
\hline
\end{tabular}

The procedure used to record the data was, in general, consistent. The TP system operators maintained logs on the operation of each of the branches on their systems. Failures and durations of failures were recorded. For most of the lines, a threshold of $1 \mathrm{~min}$ of unsatisfactory operation was used to declare a branch failed. For some of the lines, the threshold used was $10 \mathrm{~min}$.

Although the limited amount of published data on telephone line failures, such as those in [6], are not fully adequate for computing availability statistics, they have also been used for reasonableness checks on the data given here. The statistics published by common carriers tend to give higher average availabilities than those given here. The primary reason for this appears to be almost unavoidable differences in the manner in which line failure statistics are obtained. One important difference in failure statistics is the fact that branches were considered to have failed, for the purposes of this study, as soon as the system operators maintaining logs recorded failures, while the common carriers normally cannot record a failure until it is reported to them. This normally means, among other things, that short-duration self-clearing failures are not included in their database. The precise definitions of repair times used here and by the common carriers also differ in a less quantifiable manner. Hence, statistics should not be expected to agree precisely.

\section{Availability Function}

An overall probability distribution function for all 415 branches was obtained from the data. First, an availability figure was obtained for each branch by calculating the probability that the branch was operational (i.e., performing satisfactorily) during the observation period.

Each individual branch availability $A_{i}$ was calculated by

$$
A_{i}=\frac{\sum_{j=1}^{n} u_{i}}{\sum_{j=1}^{n} u_{j}+\sum_{k=1}^{m} d_{k}}
$$

where

$$
\begin{aligned}
u_{j} & =\text { branch up time in minutes } \\
d_{k} & =\text { branch down time in minutes }
\end{aligned}
$$


and summations are over all the time intervals during which the branch was observed. Another way to express this is simply

$$
A_{i}=\frac{\sum_{j=1}^{n} u_{j}}{\text { total observation time }}
$$

After calculating each branch availability, an overall availability distribution function was determined by

$$
F(A)=\operatorname{Pr}[A \leqslant a] .
$$

An overall summary curve, summarizing results for all branches observed, is shown in Fig. 2 where the probabilities are expressed in percentages for all the branches observed. The curve plotted is actually a distribution function for unavailability, one minus availability, since this gives a plot which is easier to interpret. The two curves are related by

$$
F\left(A_{C}\right)=1-F(A)
$$

with $A_{C}$ representing unavailability.

It should be noted that some of the branches were unavailable less than 0.1 percent of the time, while others were unavailable over 10 percent of the time. The total range of observed values, over three orders of magnitude base ten, indicates a very large variation in the behavior of the branches. The amount of variation is important since it reflects the uncertainty associated with the random selection of a branch. It should also be noted that 69 percent of all the branches were available 99 percent of the time or better. That is, about two thirds of the branches showed an unavailability of less than one percent.

In order to better understand the branch availability figures, the effects of several different parameters were examined. The results are discussed below.

\section{Speed Variations}

The data were separated into three speed classifications (see Fig. 3). Most (328) of the branches observed were transmitting data at 2000 or $2400 \mathrm{bits} / \mathrm{s}$ which is classified as medium speed in Fig. 3. Some of the branches (87) transmitted data at lower speeds, such as $1200 \mathrm{bits} / \mathrm{s}, 600 \mathrm{bits} / \mathrm{s}$ and lower; these lower.speed branches are grouped and classified accordingly. In addition, a curve obtained from a small sample of 11 high-speed branches (otherwise excluded from the summary data) is included for comparison purposes.

Some improvement in availability at lower speeds is indicated, but the variation within each class is more significant than the variations between classes. It is possible that a substantial percentage of the difference between availabilities observed at different speeds is due to degraded performance failures, which appear to occur more frequently at higher speeds. It is also possible that some of the improvement at lower speeds is due to less careful recording of failures in the logs of system users whose lines operated at these speeds, since these systems tended to have less critical performance requirements.

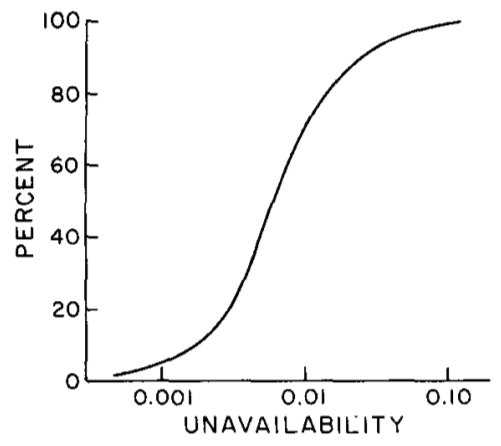

Fig. 2. Overall availability.

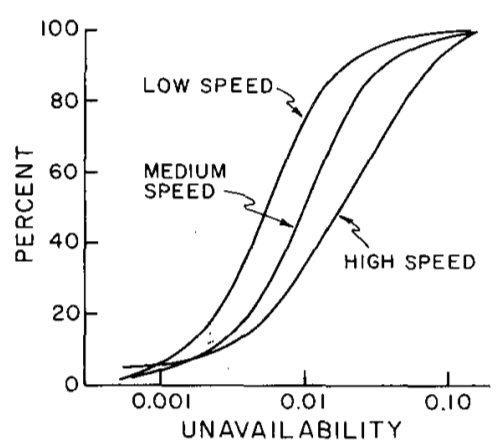

Fig. 3. Speed variations.

\section{Country and Distance Variations}

Some of the branches observed passed through more than one country. In general, they were long branches on the order of $100 \mathrm{mi}$ or more. Three classifications of branches are shown in Fig. 4. Long branches are arbitrarily classified as those longer than 50 airline mi. These long branches are separated into those within one nation, called national branches, and branches that passed through more than one country, called international branches. For contrast, branches shorter than 50 mi that existed within the boundaries of one country are also shown, even though the sample size (see Table I) is small.

Shorter branches are seen to be more reliable than longer branches. A physical interpretation of this may be that shorter branches are made up of fewer pieces of equipment, have less circuit miles of transmission, fewer weather disturbances, and involve fewer people who may inadvertently disrupt the operation. Longer branches may be subject to more of these effects. Similarly, longer branches may be thought to contain within them two or more short branch segments whose failures would be additive, thus decreasing the availability of longer branches. There is also a difference between longer branches that are international and those contained within one country. In an explanation of this, a number of factors can be cited. There are differences between the equipment in different countries, so mismatches can occur; when repair bf a failure is called for, national responsibility for the repair work may be in question, which can delay the time required to make the repair, etc.

Another set of comparison figures is shown in Fig. 5. Here two countries are compared for differences in the overall reliability of the branches. While these differences are certainly discernible, the variation within either of these countries is more significant than the variation between the countries. 


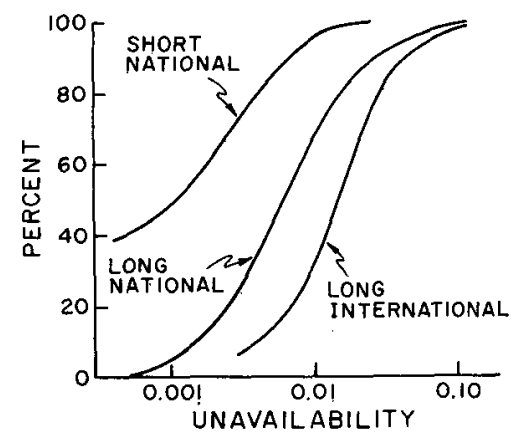

Fig. 4. National and international branches.

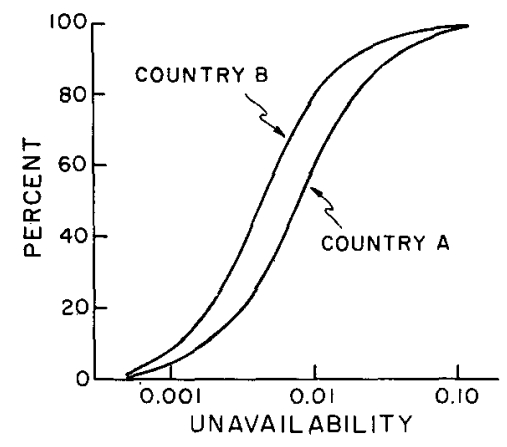

Fig. 5. Country variations.

There are, of course, errors in the recording of the data presented here. In addition, errors may also be made in interpreting the results. To help appreciate the subtle factors involved, a supplementary set of data is presented in Fig. 6. Here the same two countries are compared as in Fig. 5, but for these data, a common industry that used similar data communication systems was selected in both countries. These two curves are much more closely spaced. A way to view this is to visualize that different thresholds of acceptable system performance are imposed in different systems. Some systems are monitored more closely than others; accordingly, failures are recorded with respect to different acceptable performance levels. The reasons for these variations are too complex to elaborate on here, but they seem to exist, and they are reflected in the data. Once again, however, the variations within one country are considered more significant than the differences between the countries.

\section{Down Time Distribution}

In addition to the availability of a branch, it is important to understand the length of time a failure lasts. Given that the branch is down, a probability distribution is determined for the duration of the down time. Note, from the data, that this is a conditional probability function based on the condition that the failure has already lasted $1 \mathrm{~min}$. While failures occurred that lasted less than $1 \mathrm{~min}$, they were not included. The distribution for duration of the down time is expressed by

$$
F(D)=\operatorname{Pr}[D \leqslant t \mid D \geqslant 1 \mathrm{~min}] .
$$

This distribution function is shown in Fig. 7. Once again these probabilities are expressed as percentages. From the figure we see that some of the failures lasted longer than $10 \mathrm{~h}$

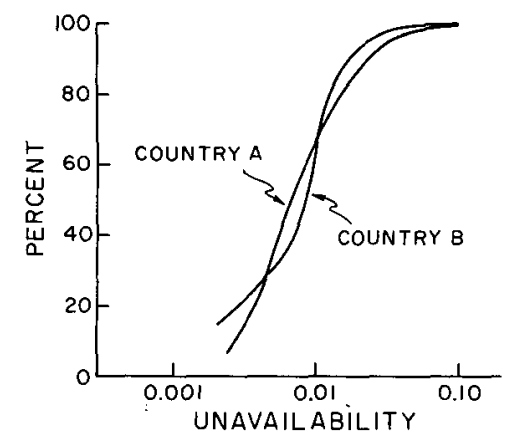

Fig. 6. Similar industry variations in two different countries.

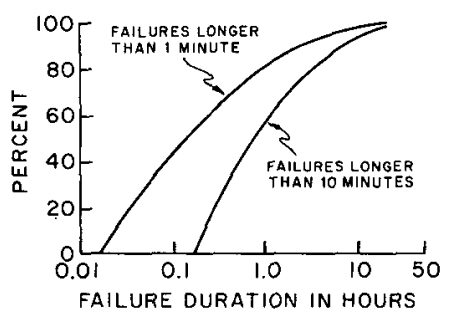

Fig. 7. Distribution of failure durations.

and 16 percent of the failures lasted longer than $1 \mathrm{~h}$. It can also be observed that 50 percent of the failures lasted less than 6 min. In addition to data for failures lasting longer than 1 min, a curve is drawn in a similar fashion for failures lasting longer than $10 \mathrm{~min}$. This was done by changing the condition and counting only failures that lasted longer than $10 \mathrm{~min}$.

One other related plot of interest is given in Fig. 8 which (very roughly) plots duration of failures versus mean time between failures (more precisely, mean time between failures of at least this duration), on a $\log -\log$ scale, for a $2400 \mathrm{bit} / \mathrm{s}$ line. Very short duration failures (or bit errors) as well as longer duration failures were considered for this plot. The plot indicates that errors affecting at least one bit ( $42 \mathrm{~ms}$ duration at $2400 \mathrm{bits} / \mathrm{s}$ ) occur roughly every $40 \mathrm{~s}$. (This corresponds to a burst error rate [3] of approximately $10^{-5}$.) Errors of at least 1 min duration occur about once per day per branch. A $1 \mathrm{~h}$ disturbance occurs approximately once each month, and disturbances lasting one day should (by extrapolating the curve slightly beyond the range for which good data are available) occur approximately once each year per branch.

The values plotted in Fig. 8 have been obtained from a variety of different sources, including [1]-[4], which reflect measurements obtained under varying conditions. This is a very general and very approximate set of data and reflects an attempt to simply summarize, and provide a rule of thumb, for understanding the overall performance of a branch selected at random and to bring within a common perspective short bit errors and longer failures which are often treated separately.

Further details of this measurement study are contained in [7]. An earlier paper by Prqyetero [8], based on a subset of the data considered here, revealed strong correlations between failures of different communication lines serving a common node, i.e., the standard assumption of independent failures for such lines was shown to be false. Theoretical analysis of failure dependencies are given in [9], [18], [19]. The more extensive data considered here have further confirmed that failures of 


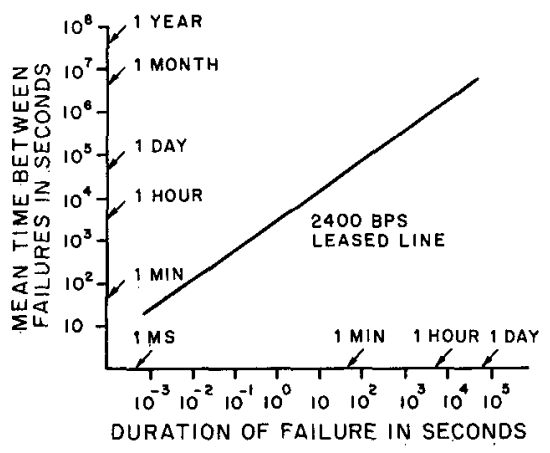

Fig. 8. Mean time between failures versus outage duration.

such lines are dependent, but better measurement data on failure dependencies could not readily be extracted from the measurements obtained.

\section{CAUSES OF VARIABILITY IN RELIABILITY PARAMETERS}

The primary causes of variability in line failure rates or similar reliability parameters appear to be the tremendous variety of equipment types and ages in the telephone plant (by far the most common source of communication links) and the correspondingly tremendous variety in operating environments for this equipment. A detailed description of the main factors causing reliability problems in the common carrier telephone plant has been given in another paper [5] .

The telephone plants in the U.S., and in many other countries, are among the most complex systems ever designed and installed by mankind. With design lifetimes for equipment of 20-40 years, and the rapid pace of technological change that has occurred within this time period, a large variety of different types and ages of equipment have been interconnected to form the installed plant. Further, operating environments for equipment range from almost ideal to cases where there are so many undesirable environmental factors (temperature variation, humidity problems, corrosive atmospheres, strong electromagnetic fields, and other factors) that it is remarkable that the equipment operates at all. Three or four orders of magnitude variation in percentage down time or failure rates for different lines or branches do not appear to be at all surprising when these factors are considered.

\section{NETWORKS CONSISTING OF MULTIPLE LINES}

Although the measurement data given here give reasonable estimates for the distribution functions for some important reliability measures for one line (recall the configuration for measurements in Fig. 1), they do not readily yield comparable data for more complex configurations consisting of multiple branches interconnected in any of a variety of ways. A heuristic model for approximating the corresponding distributions for at least the most common types of networks is given here, along with the results of a computer simulation study to verify the accuracy of the heuristic model. Additional details on the heuristic model and its validation are given in [10], [11] .

\section{Heuristic Model Introduction}

The model discussed here is a simple heuristic model for approximately computing probability distribution functions for communication system availabilities. The model assumes that failures of different branches are independent (or at least that their availabilities are independent) despite the comments given earlier about this assumption not being valid. Even the independent failure case has not been treated adequately so far, however, and extensions to handle dependent failure parameters will have to come later. All computations given here will be based on use of the overall probability distribution function in Fig. 2, since this curve is the most realistic one to assume for a general analysis.

A general formula for the availability $A$ of a system composed of a number of independently failing subsystems (with independent repairs also) can be written in the form of a sum of products of availabilities $A_{i}$ of the individual subsystems. General techniques for computing such availabilities are given in [12], [13].

The main sources of difficulty in finding percentile values for overall system availability, for reasonably complex systems, stem from the facts that percentiles are defined in terms of integrals of the appropriate probability densities and that probability laws for sums of products of random variables (even independent random variables) are normally very difficult to evaluate. (A quick verification of this can be obtained by consulting any good probability theory text, e.g., [14], for the formula giving the probability density for the product of even two independent random variables.) Some limit theorems, such as the central limit theorem, might be applicable if there is a fairly large number of terms summed; alternatively, the central limit theorem would imply that the limiting distribution for the product of a large number of independent random variables is log normal. An important constraint for the case of interest, though, is that all the random variables of interest are between zero and one (since availabilities are probabilities), and this constraint is difficult to incorporate in the limit theorems, which can be expected to converge slowly without the constraint being used. Hence, the alternative heuristic approach described below has been developed.

The analysis technique is based on first fitting a reasonable analytic curve to availability data for one branch, then approximating a corresponding analytic curve for the overall system. A reasonable analytic curve (found by trial and error) for availability of one branch is a beta density [15]:

$$
F_{A_{i}}\left(a_{i}\right)= \begin{cases}\frac{\Gamma(r+s)}{\Gamma(r) \Gamma(s)} a_{i}^{r-1}\left(1-a_{i}\right)^{s-1} & 0 \leqslant a_{i} \leqslant 1 \\ 0 & \text { elsewhere }\end{cases}
$$

with parameters $r=50$ and $s=0.5$. This density yields a mean availability of 0.990 and a 90th percentile availability of 0.973 . These values agree well with the mean and 90th percentile values of 0.990 and 0.975 , respectively, which can be computed from the data given in the previous sections of this paper or with the corresponding values of 0.988 and 0.973 , respectively, computed from [7].

The beta distribution curve [cumulative distribution curve for $1-a_{i}$ obtained from the density in (1)] is compared with the overall summary curve from Fig. 2 in Fig. 9. Although the agreement between the two curves is excellent for unavailabil- 


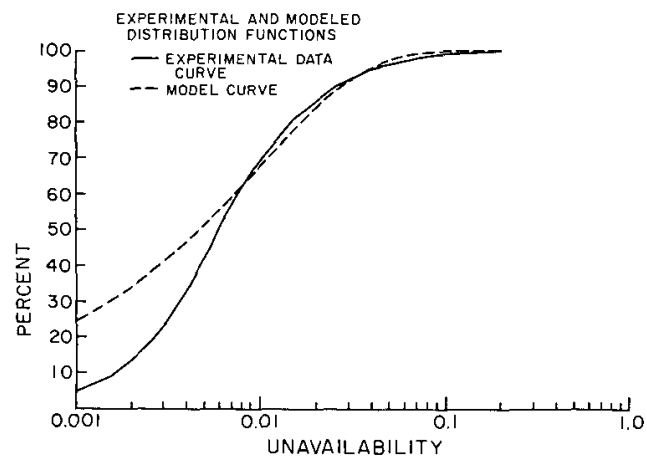

Fig. 9. Comparison of experimental and analytical unavailability distribution functions.

ities approximately above the 60th percentile, the two curves differ appreciably toward the left ends of the two plots. (The difference is greatly exaggerated by plotting the curves on a semilog scale, however; the two curves would be virtually indistinguishable on a linear scale plot.) Fortunately, the two curves agree closely for the cases of primary interest to the designer of commercial communications based systems, although different parameters chosen to improve the fit in other ranges might be advisable when working with systems, such as some military systems, with extremely high availability requirements. Extremely reliable communication lines are of little concern to many system designers, since they cause no problems for system users. The theoretical curve was chosen to give good estimates of the percentage of users of the more common commercial systems likely to obtain unsatisfactory reliability rather than to estimate how many customers would have reliability much better than they require. (It should also be noted that the measurement data are least likely to be accurate in the regions where the fit is poor, since the measurement period needed to obtain statistically significant estimates of unavailability for extremely reliable branches considerably exceeds the observation periods actually used, and the nonrigorous "statistical sampling plan" used here should not be expected to give trustworthy results for the tails of the distributions.)

The rest of this paper uses the fitted curve as if it were the true distribution function for unavailability since having an analytical formula greatly simplifies many of the computations. Later comparisons between analytical and simulation results compare the computations made with the heuristic model with simulation results obtained under the assumption the fitted curve is valid. The resulting comparisons are reasonably valid for the percentages of branches or systems likely to cause reliability problems, but they may underestimate the percentage of systems with extremely high availability.

The first and second moments of the density (6), $\overline{A_{i}}$ and $\overline{A_{i}{ }^{2}}$, are needed for the analysis below. They can be expressed in terms of the parameters $r$ and $s$ as

$$
\begin{aligned}
\bar{A}_{i} & =\frac{r}{r+s} \\
\overline{A_{i}^{2}} & =\frac{r(r+1)}{(r+s)(r+s+1)} .
\end{aligned}
$$

Alternatively, $r$ and $s$ can be expressed in terms of $\bar{A}_{i}$ and

$$
\begin{gathered}
\overline{A_{i}^{2}} \text { as } \\
r=\frac{\overline{A_{i}}\left(\overline{A_{i}}-\overline{A_{i}^{2}}\right)}{\overline{A_{i}{ }^{2}}-\left(\overline{A_{i}}\right)^{2}} \\
s=\frac{\left(1-\overline{A_{i}}\right)\left(\overline{A_{i}}-\overline{A_{i}{ }^{2}}\right) .}{\overline{A_{i}^{2}}-\left(\overline{A_{i}}\right)^{2}} .
\end{gathered}
$$

\section{Approach to Handling Complex Systems}

As has been stated earlier, techniques given in [12], [13] can be used to compute the availabilities of complex systems in terms of sums of products of availabilities of individual subsystems, but the formulas for computing probability distribution functions for functions of random variables are too complex for direct computation of the distribution functions for availabilities, even if availabilities are assumed to be independent. Reasons for eliminating the use of limit theorems from serious consideration have also been discussed. Instead, the approach adopted here is to use techniques given in [12] [13] to compute the first two moments of system availability from the first two moments for individual branches, assuming that branch availabilities are independent. Another beta distribution is then fitted to these first two moments, and this distribution is used for subsequent calculations. One rationale for this approach is that assuming a beta density for the characterization of the complex system is as logical as assuming a beta density for characterizing one branch. Simulation results confirming the validity of this approach are given later.

Computation of appropriate first and second moments for the complex system is fairly simple for cases with independent $A_{i}$ 's. The means can be computed by simply substituting mean values for each of the $A_{i}$ 's in the formula for $A$, while second moments can be readily computed (at least for cases where the formula for $A$ is reasonably simple) by utilizing the facts that second moments for products of independent random variables multiply and variances for sums of independent random variables add. These first two moments can then be used to find a new beta density to characterize the complex system by using (9) and (10) to find the parameters of this new density.

Since the availabilities of series (cascade) and parallel combinations of $N$ independent branches are given by

$$
\begin{aligned}
& A_{\text {series }}=\prod_{i=1}^{N} A_{i} \\
& A_{\text {parallel }}=1-\prod_{i=1}^{N}\left(1-A_{i}\right)
\end{aligned}
$$

the first two moments for these two simple classes of complex systems are given, for independent $a_{i}$ 's, by

$$
\begin{aligned}
& \bar{A}_{\text {series }}=\prod_{i=1}^{N} \bar{A}_{i} \\
& \bar{A}_{\text {series }}{ }^{2}=\prod_{i=1}^{N} \overline{A_{i}^{2}}
\end{aligned}
$$




$$
\begin{aligned}
& \bar{A}_{\text {parallel }}=1-\prod_{i=1}^{N}\left(1-\overline{A_{i}}\right) \\
& \bar{A}_{\text {parallel }}{ }^{2}=1-2 \prod_{i=1}^{N}\left(1-\overline{A_{i}}\right)+\prod_{i=1}^{N}\left(1-2 \overline{A_{i}}+\overline{A_{i}{ }^{2}}\right)
\end{aligned}
$$

with all terms in each product equal when the $a_{i}$ 's are identically distributed.

As [12], [13] show, most availability block diagrams for complex systems of real interest can be reduced by iterative application of the series and parallel reduction formulas, so the equations given suffice for most cases. $\mathrm{A}$ few cases where these formulas do not suffice also exist, but techniques are given in [12], [13] for handling these cases also. Although the resulting equations may, in some cases, be horribly messy, they can always be expanded to express total availability in terms of sums of products of availabilities of individual subsystems, so simple modifications of the formulas given here suffice for handling these more complex cases. The formulas given above are sufficient to handle all examples discussed in this paper.

\section{Simulation Studies}

Since the heuristic model developed here is far from rigorous, computer simulation has been used to examine the accuracy of the approach. The simulation runs were based on an assumption that the analytical curve in (1) was the true density characterizing one branch. Random variables obeying this distribution were generated to simulate availabilities of individual branches in a complex system and the rules in [12] , [13] used for determining the corresponding total system availabilities.

The beta-distributed branches were simulated using what Tocher calls the "top hat" method [16]. This involves com. puting the inverse of the cumulative distribution function so that the availability becomes the dependent variable and the probability becomes the independent variable. If the probabilities are then selected from a uniformly distributed function with values between zero and one, the corresponding availabilities follow the correct density function.

The simulation was run using some modified statistical application programs. The statistical application package used has a program for use with beta densities that computes the availability given the right tail probability. This program was modified so that it would generate a table of values for probabilities from 0.001 to 0.999 in steps of 0.001 . Uniformly distributed random numbers were generated using a standard random number generation function. All branches were assumed to have the beta distribution defined by $r=50, s=0.5$.

Systems were defined to the program as appropriate combinations of series and parallel branches. Once the system was defined, a random number was generated for each branch and the corresponding availability was selected from the tabulated data. The system availability was then calculated, for this sample, using the relationships in (10) and (11) for series and parallel combinations. (Only networks consisting of iterative combinations of series and parallel blocks have been simulated.)
The process was repeated for a number of samples specified at program run time. The cumulative distribution function was then calculated by converting the availabilities to unavailabilities and accumulating their occurrences into bins specified at run time by a "step size" declaration. The resulting distribution of availabilities is a histogram approximation to the probability density function for unavailability. To compute the cumulative distribution function, the values in the bins were simply accumulated. The resulting data were then plotted.

The statistical application package also has a program for computing right tail probabilities given the beta distribution parameters. This program was modified to generate theoretical curves shown on the same figures. The parameters of the beta distribution to be computed, $r$ and $s$, were calculated using the heuristic technique previously described.

\section{Results}

Typical comparisons between theoretical and simulation curves for six different systems are shown in Figs. 10-15. In addition, a plot of percentage error is shiown on each figure. Derived values of $r$ and $s$, maximum error between the two curves, and theoretical values for the means and 90th percentiles of availability are shown in Table II. Maximum error for any system was +6 percent. The match between simulation and heuristic model appears to be excellent, indicating that the approach gives realistic results under the assumptions that the fitted beta distribution is the true distribution characterizing one branch and that different branches fail independently. Since the agreement between the two curves in Fig. 9 is excellent for higher percentile values, the agreement with measured results for complex systems should also be good for higher percentile values (say 80th or higher percentiles) if these complex systems were composed of branches which failed independently.

The results plotted in Figs. 10-15 are directly applicable to the analysis of particular communication paths in some standard forms of networks. For example, a particular communication path in a multidrop system consists of one or more branches in cascade so Figs. 10-13 are applicable. Similar computations give path availabilities in star and loop configurations. More general networks with alternate routing may require separate computations, but the techniques are applicable.

\section{IMPLICATIONS OF RESULTS}

Since different communication lines display extreme variability in their reliability parameters (as the measurement data in Figs. 2-6 indicate), when systems are designed entirely on the basis of mean availability values, an appreciable percentage of unhappy system users is inevitable. The techniques developed here at least give approaches to estimating the percentage of unhappy users to be expected. The design can then be altered to appropriately change this percentage if this is desirable.

Both the dependent failure problem and the variable parameter problem indicate that alternative approaches should be carefully considered in lieu of relying heavily on real-time data communications in systems which require extreme reliability. 
TABLE II

TABLE OF RESULTS

\begin{tabular}{|c|c|c|c|c|c|}
\hline \multirow[b]{2}{*}{ System } & \multirow[b]{2}{*}{$r$} & \multirow[b]{2}{*}{$s$} & \multirow{2}{*}{$\begin{array}{l}\text { Maximum } \\
\text { Difference }\end{array}$} & \multicolumn{2}{|c|}{ Availability } \\
\hline & & & & Mean & 90th Percentile \\
\hline $\begin{array}{l}1 \text { branch } \\
2 \text { branches }\end{array}$ & 50.00 & 0.50 & +2 percent & 99.0 percent & 97.3 percent \\
\hline $\begin{array}{l}\text { cascaded } \\
5 \text { branches }\end{array}$ & 49.75 & 1.00 & +4 percent & 98.0 percent & 93.9 percent \\
\hline $\begin{array}{l}\text { cascaded } \\
10 \text { branches }\end{array}$ & 49.02 & 2.50 & +6 percent & 95.1 percent & 91.0 percent \\
\hline $\begin{array}{l}\text { cascaded } \\
210 \text { 's }\end{array}$ & 47.83 & 5.00 & -4 percent & 90.5 percent & 85.1 percent \\
\hline $\begin{array}{l}\text { in parallel } \\
220 \text { 's }\end{array}$ & 282.19 & 2.55 & -3 percent & 99.1 percent & 98.3 percent \\
\hline in parallel & 171.20 & 5.76 & -3 percent & 96.7 percent & 94.9 percent \\
\hline
\end{tabular}

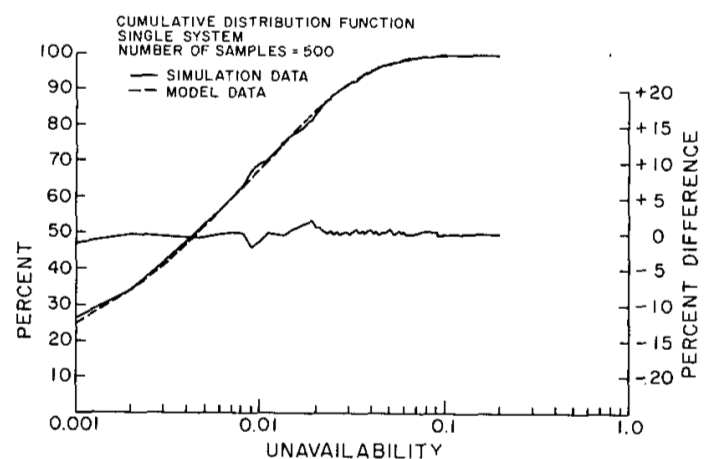

Fig. 10. Validation of simulation model for single branch.

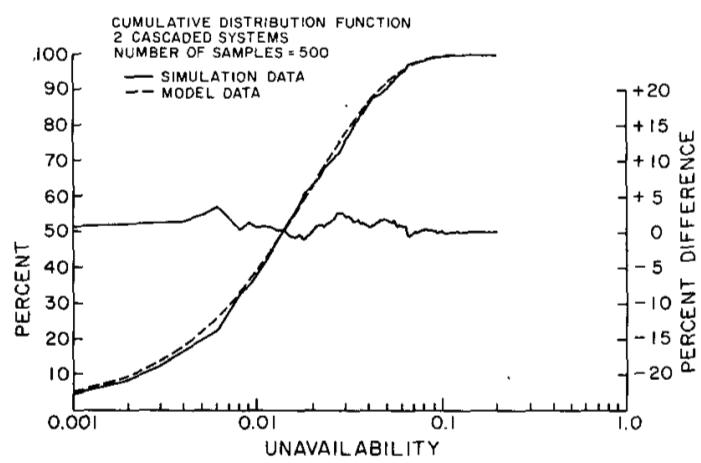

Fig. 11. Comparison between heuristic and simulation models for two cascaded branches.

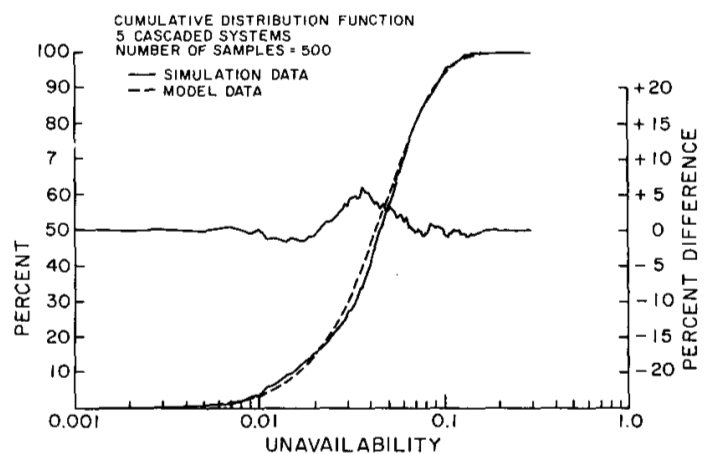

Fig. 12. Comparison between heuristic and simulation models for five cascaded branches.

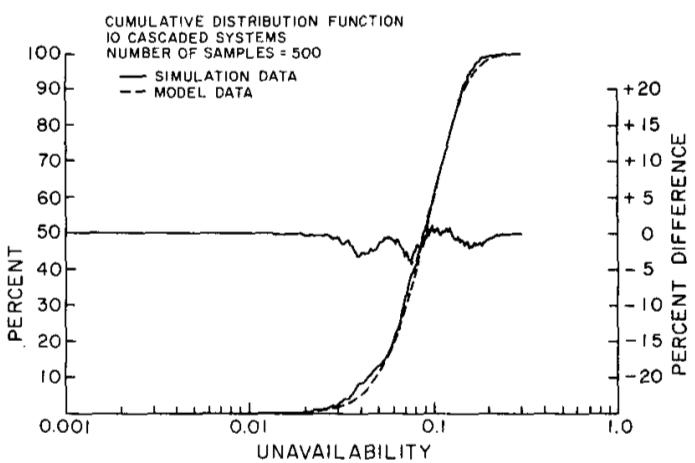

Fig. 13. Comparison between heuristic and simulation models for ten cascaded branches.

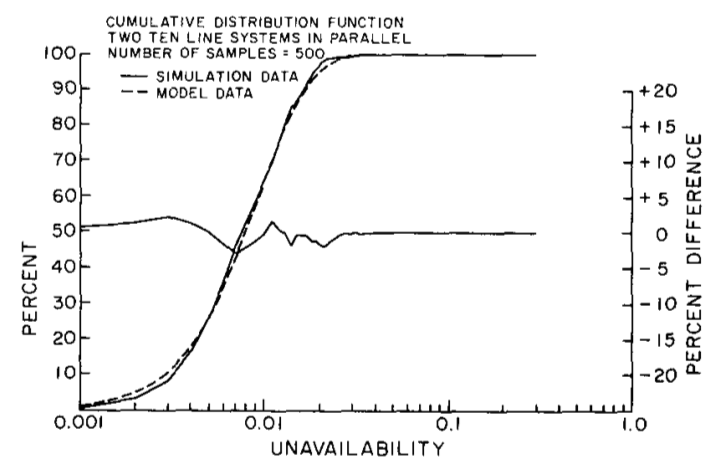

Fig. 14. Comparison between heuristic and simulation models for two ten branch systems in parallel.

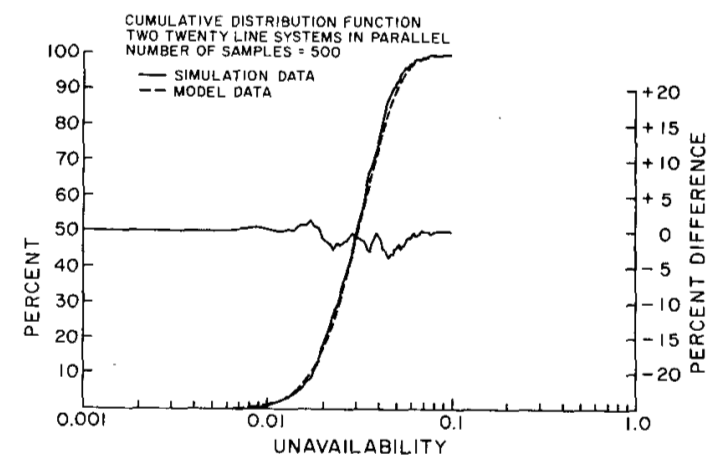

Fig. 15. Comparison between heuristic and simulation models for two twenty branch systems in parallel. 
In some cases, it may be possible to reduce such systems' dependence on real-time data communications by putting all the equipment and files needed during normal operation at one location or by providing a stand-alone mode of operation which can be used to survive communications line outages. (Both approaches, applied to operational systems, are described in [17].) Other design procedures will be better in different situations, but there are too many possibilities to attempt enumeration in this paper. One other possibility is that some systems which are desired may not be feasible.

The measurement data included here and the heuristic model for extending results to handle more complex networks consisting of multiple branches allow computation of more realistic reliability predictions than have heretofore been computable. They now make it possible to find reasonable estimates for the probability of system availability falling within certain ranges, rather than simply estimates of mean availability.

\section{EXTENSIONS TO RESEARCH}

Two primary types of extensions to this work are currently under consideration. The first is modification of the simulation runs to generate random variables obeying the measured distribution function for one branch rather than the fitted distribution function. The resulting simulation data should then be slightly more accurate, although little change in higher level percentile values is anticipated. The other modification, which is felt to be considerably more important, is to modify the techniques to at least approximately reflect the dependencies between failures of different branches. Alternative approaches to the problems, including some based on the statistical theory of extreme values, are also being considered.

Further research on developing better techniques for computing the availability of systems with dependent failures (but ignoring the variability of parameters problem) is also being pursued. New results from this research, which extend the techniques in [9] to handle more general forms of distributed networks, are given in [18], [19] .

Possibly the most obvious area where the need for further work is suggested by this paper is in obtaining better measurement data. A measurement program comparable in magnitude to the Bell System 1969-1970 connection survey [2] - [4] is needed in order to obtain truly trustworthy reliability statistics. Until such a measurement program is attempted, there will always be "a problem with the reliability of the reliability statistics offered" (quoting one of the reviewers of this paper). Hopefully, this paper may help motivate a more systematic measurement program.

\section{ACKNOWLEDGMENT}

The authors wish to acknowledge the efforts of the many people who conscientiously collected the data used in this paper. In particular, thanks go to M. A. Berk, J. Provetero, and A. Jama of IBM Corporation who assembled and organized various parts of this information into a useful description. Also, thanks go to B. O. Evans and E. H. Sussenguth of IBM for their support in doing this work.

\section{REFERENCES}

[1] A. A. Alexander, R. M. Gryb, and D. W. Nast, "Capabilities of the telephone network for data transmission," Bell Syst. Tech. J. vol. 39, pp. 431-476, May 1960.

[2] F. P. Duffy and T. W. Thatcher, Sr., " 1969-70 connection survey: Analog transmission performance on the switched telecommunications network," Bell Syst. Tech. J., vol. 50, pp. 1311-1347, Apr. 1971.

[3] M. D. Balkovic, H. W. Klancer, S. W. Klare, and W. G. McGruther. "1969-70 connection survey: High-speed voiceband data transmission performance on the switched telecommunications network," Bell Syst. Tech. J., vol. 50, pp. 1349-1384, Apr. 1971.

[4] H. C. Fleming and R. M. Hutchinson, Jr., " 1969-70 connection survey: Low speed data transmission performance on the switched telecommunications network," Bell Syst. Tech. J., vol. 50, pp. 1385-1405, Apr. 1971.

[5] J. Spragins, "Data transmission over the common carrier telephone plant: Factors affecting its reliability," in Conf. Rec. 1978 Int. Conf. Commun., Toronto, Ont., Canada, June 1978, pp. 3.1.13.1.5.

[6] Bell Syst. Tech. Ref., "Data communications using voiceband private line channels,"' PUB41004, Oct. 1973.

[7] J. D. Markov, M. W. Doss, and S. A. Mitchell, " A reliability model for data communications," in Conf. Rec. 1978 Int. Conf. Commun., Toronto, Ont., Canada, June 1978, pp. 3.4.1-3.4.5.

[8] J. Provetero, "Availability of voice grade private wire telephone lines," in Proc. IEEE Fall Electron. Conf., Chicago, IL, Oct. 1971 , pp. 392-397.

[9] J. Spragins, "Dependent failures in data communication systems," IEEE Trans. Commun., vol. COM-25, pp. 1494-1498, Dec. 1977.

[10] J. Spragins and D. Squire, "Data communication network reliability calculations with real world distributions for reliability parameters," in Proc. 1979 Comput. Networking Symp., Gaithersburg, MD, Dec. 1979, pp. 110-116.

[11] D. Squire, "A simulation study of a heuristic technique for approximating availability percentiles for cascaded independent systems," M.S. thesis, Oregon State Univ.. Corvallis, Dec. 1978.

[12] J. A. Buzacott, "Finding the MTBF of repairable systems by reduction of the reliability block diagram," Microelectron. Rel., vol. 6 , pp. 105-112, 1967.

[13] _ _ "Network approaches to finding the reliability of repairable systems," IEEE Trans. Rel., vol. R-19, pp. 140-146, Nov. 1970.

[14] A. Papoulis, Probability, Random Variables, and Stochastic Processes. New York: McGraw-Hill, 1965.

[15] S. S. Wilks, Mathematical Statistics. New York: Wiley, 1962.

[16] K. D. Tocher, The Art of Simulation. London, England: English Univ. Press, 1963.

[17] R. O. Hippert, L. R. Palouneck, J. Provetero, and R. O. Skatrud, "Reliability, availability and serviceability design considerations for the supermarket and retail store systems," IBM Syst. J., vol. 14, no. 1, pp. 81-95, 1975.

[18] J. Spragins and J. Assiri, "Communication network reliability calculations with dependent failures," in Conf. Rec. $1980 \mathrm{Nat}$. Telecommun. Conf. Houston, TX, Dec. 1980, pp. 25.2.1-25.2.5.

[19] J. Assiri, "Development of dependent failure reliability models for distributed communication networks." Ph.D. dissertation, Oregon State Univ., Corvallis, June 1980.

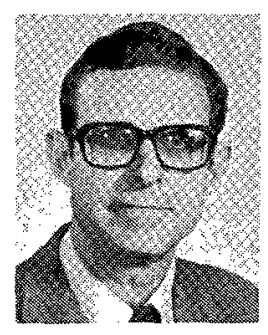

John D. Spragins (S'56-M'59) received the B.S. degree in electrical engineering from Oklahoma State University, Stillwater, in 1956 and the M.S. and Ph.D. degrees in electrical engineering from Stanford University, Stanford, $\mathrm{CA}$, in 1958 and 1964 , respectively

Since 1980 he has been a Professor of Electrical and Computer Engineering at Clemson University, Clemson, SC. Prior to this he was an Associate Professor at Oregon State University, Corvallis, Research Staff Member and Advisory Engineer at IBM Corporation, Research Triangle Park, NC, Principal Engineer at General Electric Computer Equipment Division, Phoenix, $\mathrm{AZ}$, and Assistant Professor at Arizona State University, Tempe. His 
current research interests include developing improved reliability and performance models for computer communications networks, new network configurations, and networking protocols. He has published over 40 reports and papers in these and allied fields.

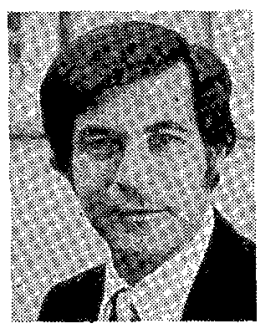

James D. Markov received the B.S. degree in electrical engineering from the University of Akron, Akron, $\mathrm{OH}$, in 1961, and the M.S. degree in engineering sciences from the University of Alabama, Tuscaloosa, in 1970.

$\mathrm{He}$ is a Senior Engineering Manager with IBM's Systems Communication Division, Research Triangle Park, NC. He is currently the Manager of System Projects for Local Networks. Prior to his current position, he was Manager of Networking Architecture, a position he held from 1975 to 1978. Since 1970 he has been working in the field of communications system architecture with a major emphasis on the development of SNA. During the 1960's he worked on the development of the guidance and control system for the Saturn vehicle as part of the Apollo program.

Mr. Markov is a member of Sigma Tau.
M. W. Doss, photograph and biography not available at the time of publication.

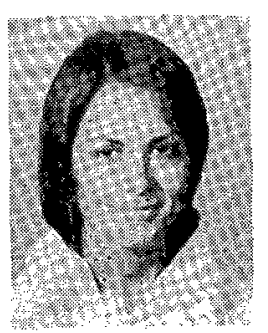

Stephanie A. Mitchell was born in Greensboro. NC. She received the B.S. degree in mathematics (magna cum laude) from Wake Forest University. Winston-Salem, NC, in 1972.

She joined IBM in its Systems Communications Division in 1972. In 1976 she attended IBM's Systems Research Institute and transferred to IBM's Data Processing Division. WinstonSalem. in 1977.

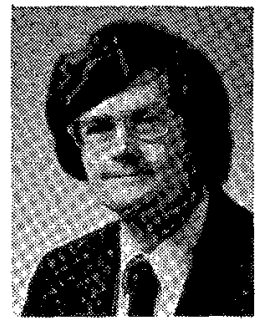

David C. Squire received the B.S.E.E. and M.S.E.E. degrees from Oregon State University, Corvallis, in 1967 and 1979, respectively. through a joint program offered by Tektronix, Inc. and Oregon State University.

He worked at NASA's Ames Research Center as a Research Engineer from 1967 to 1969 and joined Tektronix, Inc., Beayerton, OR, in 1969 where he is now Manager of the Raster Scan Terminal Development Group. 